\title{
XXXVI.-A Reaction of certain Colouring Matters of the Oxazine Series
}

By Jocelyn Field Thorpe.

DURING some experiments on the staining of pathological sections for the microscope, Professor Lorrain Smith and Dr. Powell White, of the Manchester University, made the discovery that certain blue colouring matters of the oxazine series, when used as a stain for sections containing neutral fat globules, possessed the remarkable property of colouring the fatty matter red, while, in the ordinary course, staining the protein matter blue. The two colouring matters of the series which they found exhibited this property most strongly were Nileblue A (1) and New Methylene-blue GG (2), although Nile-blue BB (3) and Meldola's blue (4) also gave the same reaction after having been previously boiled with very dilute mineral acid.

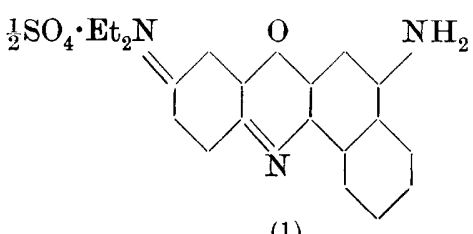

(1).

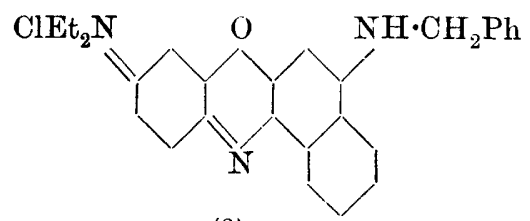

(3).

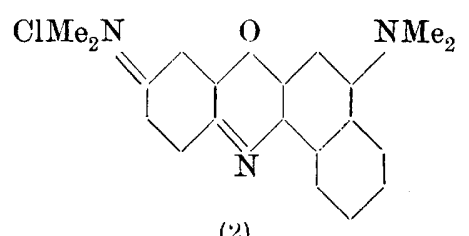

$(2)$.

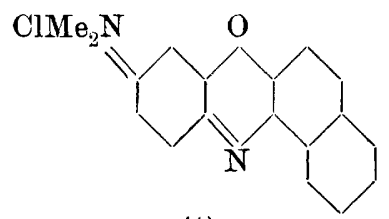

(4).

These gentlemen also found that the compound producing the red fat stain could be extracted from the blue solution of the colouring matter by means of xylene, in which it formed a red solution showing strong yellow fluorescence, and that after being completely extracted in this way more could be obtained on warming the dye solution with dilute bydrochloric or sulphuric acids. At their request I undertook to investigate the chemistry of this phenomenon.

Experiments with several typical dye-stuffs of the oxazine series showed at once that this reaction was exhibited only by those colouring matters which are derivatives of phenonaphthoxazine (5), and that those dyes which are derivatives of phenoxazine (6) were not capable of 
being converted into a red compound under the same experimental conditions.<smiles></smiles>

(5).<smiles>O=C1CCCCC1NC1CCCCC1</smiles>

(6).

Thus the two Capri-blues GN (7) and GON (8) did not give this reaction, which was, however, exhibited by New-blue B (Cassella) (9),

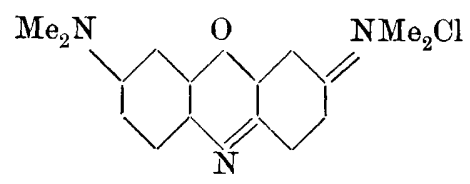

(7).

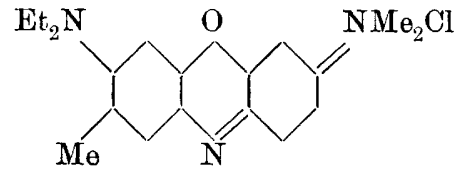

(8).

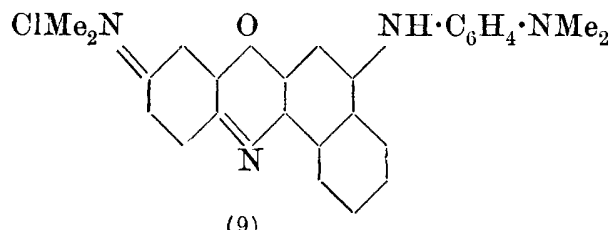

(9).

as well as by those colouring matters the formulæ of which are given on page 324. The oxazine colouring matters which are capable of being converted into a red compound can therefore be divided into two classes. (a) Those derivatives of phenonaphthoxazine which contain an amino- or substituted amino-group in the position 6.* b) Those which have this position free. Of class $(a)$, Nile-blue A (1), Nile-blue BB (3), New Methylene-blue GG (2), and New-blue (B) (9) are instances, whereas the only commercial colouring matter which falls into class (b) is Meldola's blue (4).

In the case of dye-stuffs of class $(a)$, the production of the red compound is due to the replacement of the amino- or substituted amino-group by hydroxyl and the consequent formation by intermolecular change (see p. 331) of the corresponding phenonaphthoxazorie. Thus Nile-blue A (1) and Nile-blue BB (3) are converted into 3-diethylaminophenonaphthoxazone (10), whereas New Methyleneblue GG (2) and New-blue B (9) are converted into 3-dimethylaminophenonaphthoxazone (11).

* The method of numbering is that recommended by Kehrinann and Gauhe (Ber. 1897, 30, 2131).

VOL. XCI. 


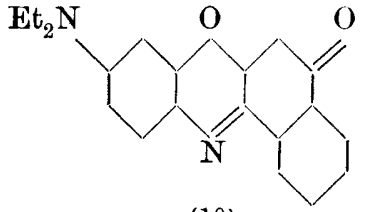

(10).

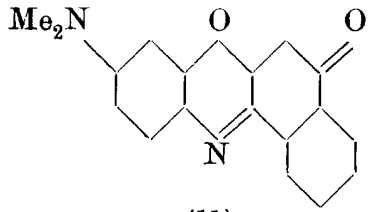

(11).

Both these compounds have been prepared by Möhlau and Uhlmann, the former (Annalen, 1896, 289, 124) by the interaction of nitrosodiethyl- $m$-aminophenol and $\alpha$-naphthol, and the latter (loc. cit., p. 126) from nitrosodimethyl- $m$-aminophenol and $\alpha$-naphthol. The compounds prepared from the colouring matters mentioned above are identical with these substances described by Möhlau and Uhlmann.

The rapidity with which the colouring matters of Class $(a)$ are converted into the oxazones is dependent on the condition of the aminogroup in the position 6. If this group is unsubstituted, as is the case in Nile-blue A (1), its replacement takes place remarkably quickly, and an aqueous solution of 3 grams of the blue can be completely converted into the oxazone on heating on the water-bath for twelve hours, provided that the solution is rendered slightly acid by means of dilute sulphuric acid and that it is covered with some suitable solvent such as xylene, which will extract the oxazone as soon as it is formed.

No trace of diethylaminophenonaphthoxazone (10) can be extracted from the solid colouring matter, and it is apparently first formed on dissolving the dye in water. The quantity, however, produced in this way is extremely small, but owing to the fluorescence exhibited by its solutions, its presence can be readily detected by shaking the dye solution with ether or xylene.

If the amino-group is substituted by alphyl groups, as is the case in Now Methylene-blue (2), its replacement takes place almost as quickly as when it is unsubstituted. Thus a solution of 3 grams of New Methylene-blue can be completely converted into dimethylaminophenonaphthoxazone (11) on heating on the water-bath for fifteen hours, the conditions being the same as those indicated in the case of Nile-blue A. If, however, the amino-group is substituted by benzyl (Nile-blue BB) or by the dimethylaminophenyl group (New-blue B), its replacement takes place with much greater difficulty, and it is necessary to heat an acidified solution of the dye for several days before the whole of it will have been transformed into the oxazone.

Both dimethylaminophenonaphthoxazone (11) and diethylaminophenonaphthoxazone (10) form salts with hydrochloric acid containing one equivalent of the acid. These salts form deep blue stable solutions in hot dilute hydrochloric acid, but are dissociated by water. 
It is probable therefore that they possess the formulæ (12) and (13) respectively,

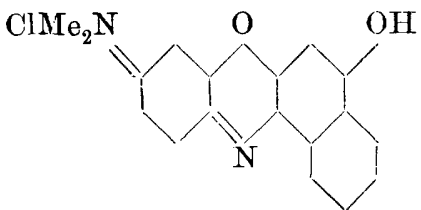

(12).

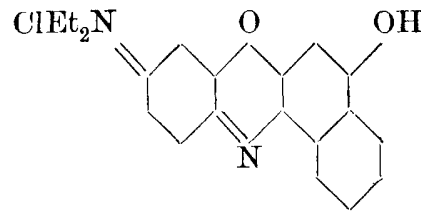

(13).

and that the passage of the salt to the base is accompanied by intermolecular rearrangement. In any case, if the $p$-quinone formula for these dyestuffs is correct, compounds of formulæ (12) and (13) must occur as intermediate products in the transformation of Nile-blue A. and New Methylene-blue $G G$ into the corresponding oxazones. If, however, the oxonium formula of Kehrmann is employed, the assumption of an intermolecular change is unnecessary. Thus, in the case of New Methylene-blue, the change can be represented by the formulw

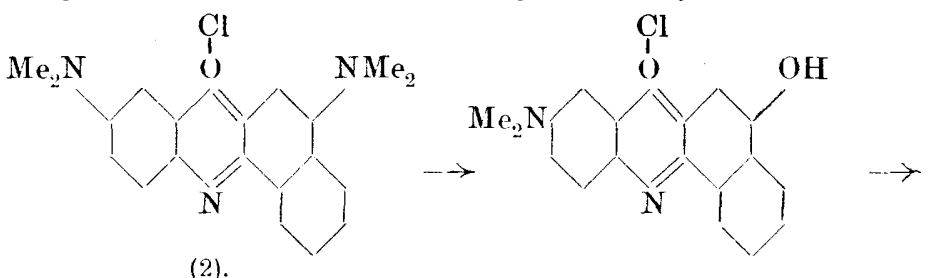

(2).

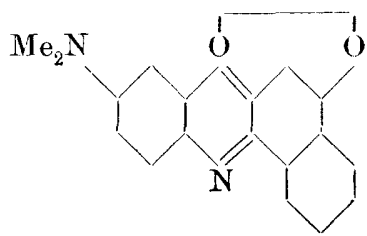

It should be mentioned, nevertheless, in connexion with this point, that 2-aminophenonaphthoxazone (14), which Kehrmann and Gauhe (Ber., 1897, 30, 2132) prepared from the corresponding 2-nitroderivative, and which is precluded from forming a $p$-quinone salt, although not from yielding a salt of oxonium, dissolves in dilute hydrochloric acid, forming a yellow solution,

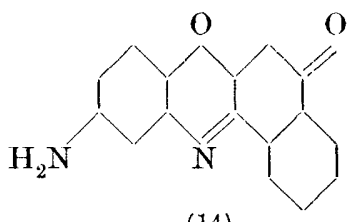

(14).

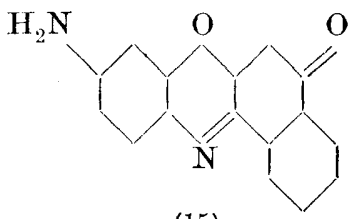

(15).

whereas 3-aminophenonaphthoxazone (15), also prepared by Kehrmann and Gauhe (loc. cit., 2136), in which the formation of a $p$-quinone salt 
is possible, gives violet-blue solutions in dilute mineral acids, or, in other words, behaves in the same way as the 3-dialkylaminophenonaphthoxazones described above.

There can be no doubt that the blue salts of the dialkylaminophenonaphthoxazones remain dissolved in the dye solutions from which they are derived; at any rate, a considerable proportion of the oxazine colouring matter can be converted into the salt of the oxazone without any dissociation of this salt taking place. This can be readily shown by taking advantage of the fact that if wool is boiled in a hot acidified solution of the salt of the oxazone it is dyed a deep blue, which, owing to the decomposition of the salt on the fibre, is changed to red on washing with water. Nile-blue $A$ can be boiled for a considerable time with dilute acid without any appreciable change in colour taking place, but if wool is dyed in the boiled solution of the blue, a dyed fabric will be obtained which will become markedly purple on washing with water; moreover, the presence of the oxazone in large amount in the boiled dye solution can be illustrated by the fact that when shaken with xylene the xylene solution becomes strongly fluorescent.

Xylene therefore has the power of extracting the weak oxazone base from a solution of its salt, and there can be no doubt that the mechanism of the reaction by which the neutral fat globules are stained red is of the same kind. The fat, in which the oxazone is readily soluble, extracts the base from the solution of its salt, becoming thereby coloured a brilliant red; at the same time the protein matter in the section is coloured blue by the excess of unchanged Nile-blue present.

The only commercial colouring matter belonging to Class $(b)$ is Meldola's blue (4), which does not give the red fat stain until it has been boiled for some time with dilute acid. In this case also the compound producing the red stain can be extracted by means of xylene. When isolated, this substance was found to be identical with the compound produced from New Methylene-blue, or, in other words, to be 3-dimethylaminophenonaphthoxazone (11):

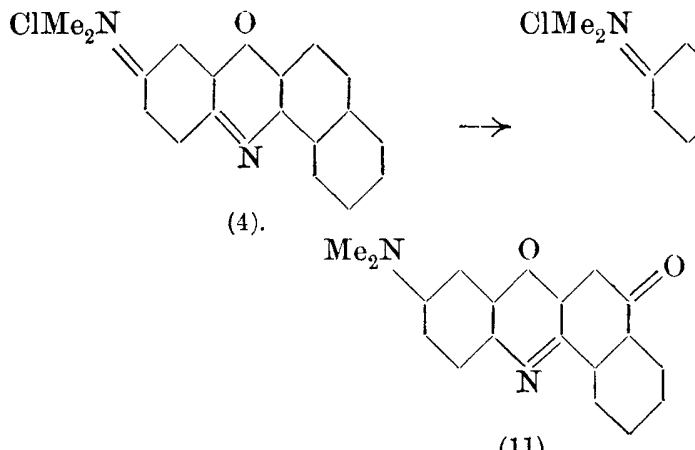

(11). 
In this case, therefore, the entrance of an hydroxyl group has been caused to take place in the position 6 .

On searching the literature for other instances of this rather remark able reaction, it was found that Fischer and Hepp (Ber., 1903, 36, 1810) had recorded a similar reaction in the case of phenonaphthoxazone (16), which they found on boiling in alcoholic solution with hydrochloric acid to be partially transformed into 3-hydroxyphenonaphthoxazone (17).

They explain this reaction by assuming that water is first added on to the quinone ring, forming an oxime, from which the phenonaphthoxazone is produced by intermolecular change; the two superfluous hydrogen atoms being removed by the oxygen of the air thus :
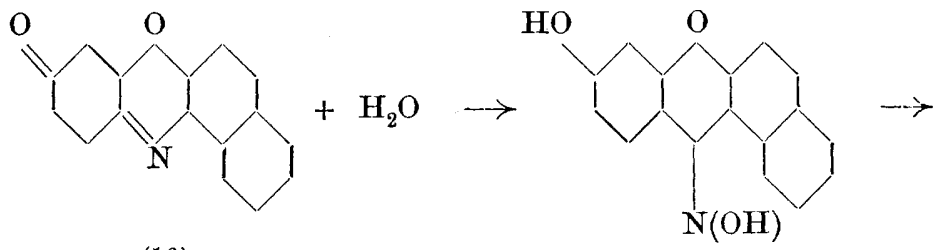

(16).

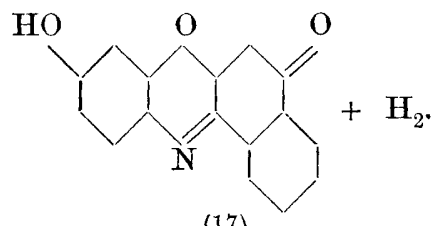

(17).

In the case of Meldola's blue it is probable that these hydrogen atoms reduce a portion of the colouring matter to its leuco-base. Strong evidence was obtained of the formation of this base, although it was not found possible to isolate it in a condition sufficiently pure for analysis. The leuco-base, however, readily oxidises in the air, regenerating the base of the colouring matter, and therefore if precautions are taken to allow of the free access of air from time to time during the course of the experiment, about 80 per cent. of a weighed quantity of Meldola's blue can be converted into the oxazone.

Möhlau and Uhlmann (Annalen, 1896, 289, 120) prepared the base both of 3-dimethylaminophenonaphthoxazine chloride (Methyl Nileblue) and of 3-diethylaminophenonaphthoxazine sulphate (Ethyl Nileblue, Nile-blue A), and describe these compounds as forming strongly fluorescent solutions in organic solvents. These substances, which in their anhydrous state have the formulæ (18) and (19) respectively, 


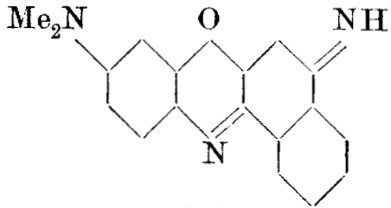

(18).

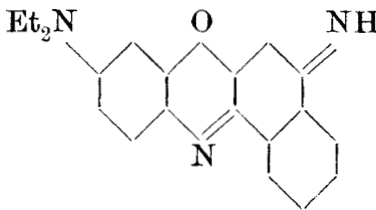

(19).

were prepared from the solution of the colouring matter by the action of caustic soda. The base of Nile-blue A according to my experiments does not form solutions which are fluorescent in ordinary daylight, and it is only when it is mixed with the corresponding oxazone that such fluorescent solutions are produced.

Experiments show that it is impossible to obtain the base of the colouring matter in the manner described by these chemists without it being mixed with varying quantities of the oxazone. Möhlan and Uhlmann obtained the base in pale brown needles from hot dilute alcohol, a process in itself probably sufficient to convert some of it into the oxazone. Moreover, they only estimated the percentage of carbon and hydrogen in their product, whicb, since the difference in the percentage of these elements in the oxazone and oxazine is within the limits of experimental error, is not sufficient to distinguish between them. A nitrogen determination would have indicated that they were working with a mixture of the two compounds.

That the base of Nile-blue A forms non-fluorescent solutions is shown by the following experiment. An acidified solution of the colouring matter was extracted with xylene until the extract ceased to be coloured, when it was cautiously treated with dilute caustic soda solution and again extracted with xylene. The insoluble base which was precipitated on the addition of the alkali dissolved in the xylene, imparting to it a deep red colour without fluorescence. The xylene solution was carefully dried and then concentrated by distillation, the residue after being freed from the remainder of the xylene on the water-bath being crystallised from benzene. The pale brown needles obtained in this way were dried at $100^{\circ}$ to constant weight and analysed:

$0 \cdot 2307$ gave $26 \cdot 3$ c.c. of nitrogen at $17^{\circ}$ and $765 \mathrm{~mm}$. $\quad \mathrm{N}=13.43$.

$$
\mathrm{C}_{20} \mathrm{H}_{19} \mathrm{ON}_{3} \text { requires } \mathrm{N}=13 \cdot 3 \text { per cent. }
$$

The base dissolves in most of the usual organic solvents, forming reddish solutions without fluorescence, all the other properties of the compound being the same as those described by Möhlau and Uhlmann. It differs from the oxazone in being completely extracted from its xylene solution by means of dilute hydrochloric acid, whereas a solution of the oxazone can be shaken with dilute acid without any of it being extracted. In this way the presence of the oxazone in the base 
prepared according to the directions of Möhlau and Uhlmann was established.

\section{Formation of 3-Diethylaminophenonaphthoxazone from Nile-blue $A$ and from Nile.blue $2 B$.}

Nile-blue A.-In the first instance the solid commercial colouring matter was extracted with ether and with xylene. In both cases the solvent remained colourless, showing that the oxazone was not present ; in order, however, to remove any impurities a quantity of the colouring matter was crystallised from water and the amount of nitrogen determined in the product:

0.2154 gave 19.6 c.c. of nitrogen at $17^{\circ}$ and $770 \mathrm{~mm}$. $\quad \mathrm{N}=11.63$, $\left(\mathrm{C}_{20} \mathrm{H}_{20} \mathrm{ON}_{3}\right)_{2}, \mathrm{SO}_{4}$ requires $\mathrm{N}=11 \cdot 5$ per cent.

Three grams of the purified dye were dissolved in 500 c.c. of water contained in a large flask, 3 c.c. of concentrated sulphuric acid added, and the solution mixed with 100 c.c. of xylene. On shaking the flask vigorously a considerable amount of red colouring matter passed into solution in the xylene, imparting to it a characteristic yellow fluorescence. The flask was then heated on the water-bath for twelve hours, being constantly shaken during the period of heating. The colour of the xylene gradually increased in intensity, and at the end of the time named had become deep red with strong yellow fluorescence; at the same time the colour of the dye solution became weaker, and at the end of the twelve hours had practically disappeared. The xylene solution was then separated and concentrated by distillation, the residuo being poured into an evaporating dish and freed as far as possible from xylene by heating on the water-bath. The residue, which solidified on cooling, was washed with a little ether to free it from the last traces of xylene, filtered, and crystallised from light petroleum (b. p. $80-100^{\circ}$ ). In this way the oxazone was obtained in brown glistening laminæ, with faint green metallic reflex, melting at $205^{\circ}$ :

0.2009 gave 15.2 c.c. of nitrogen at $24^{\circ}$ and $765 \mathrm{~mm} . \quad \mathrm{N}=8.63$. $\mathrm{C}_{20} \mathrm{H}_{18} \mathrm{O}_{2} \mathrm{~N}_{2}$ requires $\mathrm{N}=8.8$ per cent.

The properties of the oxazone correspond in every way with those of 3-diethylaminophenonaphthoxazone, described by Möhlau and Uhlmann.

The hydrochloride, $\mathrm{C}_{20} \mathrm{H}_{18} \mathrm{O}_{2} \mathrm{~N}_{2}, \mathrm{HCl}$, was prepared by boiling the oxazone with dilute hydrochloric acid and allowing the deep blue solution to cool, the dark blue crystalline precipitate which then separated being isolated by filtration and dried in an evacuated desiccator. It was analysed by boiling a weighed quantity with water until the 
filtrate was colourless, filtering, and estimating the amount of hydrochloric acid in the filtrate :

$$
\begin{aligned}
& 0.2103 \text { gave } 0.0858 \mathrm{AgCl} . \quad \mathrm{Cl}=10 \cdot 13 . \\
& \qquad \mathrm{C}_{20} \mathrm{H}_{18} \mathrm{O}_{2} \mathrm{~N}_{2}, \mathrm{HCl} \text { requires } \mathrm{Cl}=10 \cdot 1 .
\end{aligned}
$$

Nile-blue 2B.-The solid commercial dyestuff when warmed with ether or xylene imparted no colour to the solvent, neither did a solution of it when shaken with these liquids give any indication of the presence of the oxazone. A quantity was purified by crystallisation from water and analysed :

$$
\begin{aligned}
& 0.2210 \text { gave } 17.9 \text { c.c. of nitrogen at } 18^{\circ} \text { and } 771 \mathrm{~mm} \text {. } \mathrm{N}=9.62 . \\
& \qquad \mathrm{C}_{27} \mathrm{H}_{25} \mathrm{ON}_{3}, \mathrm{HCl} \text { requires } \mathrm{N}=9.5 \text { per cent. }
\end{aligned}
$$

Six grams of the purified colouring matter were dissolved in 1000 c.c. of water, and after being mixed with 10 c.c. of concentrated hydrochloric acid and 200 c.c. of xylene heated on the water-bath, with constant shaking, for twenty-four hours. During the course of heating the upper layer of xylene gradually became intensely fluorescent, but at the end of the time named the dye solution still remained strongly coloured. The xylene solution was therefore removed by aid of the separating funnel and the blue solution of the dye mixed with 100 c.c. of fresh xylene, and the heating continued. Meanwhile the xylene solution from the first extraction was concentrated by distillation, and the residue, after being freed from xylene on the water-bath, washed with ether and crystallised from light petroleum (b. p. $80-100^{\circ}$ ).

The oxazone prepared in this way was in every way identical with the compound derived from Nile-blue A :

0.2517 gave 18.3 c.c. of nitrogen at $16^{\circ}$ and $768 \mathrm{~mm}$. $\quad \mathrm{N}=8.64$.

$$
\mathrm{C}_{20} \mathrm{H}_{18} \mathrm{O}_{2} \mathrm{~N}_{2} \text { requires } \mathrm{N}=8 \cdot 8 \text { per cent. }
$$

The quantity obtained from this experiment was about 0.5 gram. The dye solution which had been mixed with a further quantity of xylene was heated for twenty-four hours longer, when a further amount of about $0.5 \mathrm{gram}$ of the oxazone was obtained on evaporating the xylene solution. Fresh xylene was then again added and the heating continued, the process being repeated until the solution of the dye had become colourless, an operation which required 96 hours in all.

The aqueous solution from these experiments was evaporated on the water-bath to a small bulk, filtered, and the filtrate rendered alkaline by means of dilute caustic soda solution. The oil which then separated was extracted by means of ether, the ethereal solution dried, and freed from ether by evaporation. The small quantity of residual oil boiled at $185^{\circ}$ and the following analysis showed it to be benzylamine:

0.2103 gare $0.6037 \mathrm{CO}_{2}$ and $0.1607 \mathrm{H}_{2} \mathrm{O} . \mathrm{C}=78.32 ; \mathrm{H}=8.49$. $\mathrm{C}_{7} \mathrm{H}_{9} \mathrm{~N}$ requires $\mathrm{C}=78.5 ; \mathrm{H}=8 \cdot 4$ per cent. 


\section{Formation of 3-Dimethylaminophenonaphthoxazone from New Methylene- blue $G G$ and from New-blue $B$.}

Methylene-blue GG.-The specimen of this colouring matter was obtained from Dr. Grübler, of Leipzig, and had been specially purified for pathological work. It was shown to be pure by the following analysis :

$0 \cdot 2010$ gave $20 \cdot 6$ c.c. of nitrogen at $18^{\circ}$ and $769 \mathrm{~mm}$. $\quad \mathrm{N}=12 \cdot 12$. $\mathrm{C}_{20} \mathrm{H}_{19} \mathrm{ON}_{3}, \mathrm{HCl}$ requires $\mathrm{N}=11.9$ per cent.

In the first instance the pure dyestuff was extracted with xylene in order to ascertain whether any of the oxazone was present. Since the xylene did not become coloured even on warming it was apparent that the solid dye did not contain any; 3 grams were therefore dissolved in 500 c.c. of water, 5 c.c. of concentrated hydrochloric acid added, and the mixture covered with 100 c.c. of xylene. The xylene solution became strongly coloured on vigorously shaking the flask, showing that the oxazone had commenced to be formed as soon as the colouring matter had dissolved in water. The mixture was heated on the water-bath for fifteen hours, the flask being constantly shaken throughout the time of heating. The xylene solution became gradually deeper in colour as the reaction progressed, and the colour of the dye solution became gradually weaker, until at the end of the fifteen hours the xylene had hecome deep red in colour with strong yellow fluorescence and the colour of the dye solution had practically disappeared. The layer of xylene was separated and freed from xylene first by distillation and finally by evaporation in an open basin on the water-bath. The solid product when crystallised from xylene was obtained in long, dark coloured needles, appearing brown by transmitted light, which melted at $244^{\circ}$ to a red liquid with green metallic reflex :

0.2071 gave 17.0 c.c. of nitrogen at $15.5^{\circ}$ and $777 \mathrm{~mm} . \quad \mathrm{N}=9.85$.

$$
\mathrm{C}_{18} \mathrm{H}_{14} \mathrm{O}_{2} \mathrm{~N}_{2} \text { requires } \mathrm{N}=9 \cdot 7 \text { per cent. }
$$

The oxazone, which is in all respects identical with the compound prepared by Möhlau and Uhlmann, dissolves in hot dilute hydrochloric acid, forming a deep blue solution from which the hydrochloride separates on cooling in dark blue needles with metallic reflex. The salt, which is dissociated by water, was analysed by boiling a weighed quantity with water, filtering, and estimating the amount of hydrogen chloride in the filtrate :

0.2171 gave $0.0948 \mathrm{AgCl}$. $\mathrm{Cl}=10 \cdot 81$.

$$
\mathrm{C}_{18} \mathrm{H}_{14} \mathrm{O}_{2} \mathrm{~N}_{2}, \mathrm{HCl} \text { requires } \mathrm{Cl}=10 \cdot 9 \text { per cent. }
$$

New-blue B.-This colouring matter, which for the purposes of this research was obtained from Messrs. Cassella \& Co. of Frankfort-am- 
Main, has recently formed the subject of a communication by J. Formánek in a paper dealing with the spectroscopic investigation of the colouring matters of the oxazine series (Zeitsch. Furb. Ind., 1907, 6, 1). The dyestuff is prepared by the action of asym-dimethyl-p-phenylenediamine on Meldola's blue, and Formánek finds, as a result of the study of the absorption spectra given by a dilute solution, that it is always admixed with a quantity of unchanged Meldola's blue. It was noticed that the dyestuff afte $r$ repeated recrystallisation did not aprear to be a homogeneous substance and that the percentage of nitrogen in the recrystallised product always gave a value too low for a compound corresponding with the constitution assigned to New-blue B. It was therefore not found possible to prepare a pure specimen of the colouring matter, and since Meldola's blue also gives 3-dimethylaminophenonaphthoxazone on boiling with dilute acids, it was difficult to prove conclusively that the oxazone had been formed by the elimination of the dimethyl-p-phenylenediamine residue from New-blue B.

The point was, however, settled by the actual isolation of asym-dimethyl-p-phenylenediamine from the mother liquors after the conversion of the colouring matter into the oxazone.

The process adopted was similar to that used in the previous cases. Six grams of the blue were dissolved in 1000 c.c. of water and, after being mixed with 10 c.c. of concentrated hydrochloric acid and 200 c.o. of xylene, heated on the water-bath, with constant shaking, for twelve hours. After this time the strongly coloured xylene solution was separated and the dye solution again mixed with xylene and heated for a further twelve hours. The xylene solution was then once more separated and the aqueous solution mixed with fresh xylene and again heated for twelve hours. After this process had been repeated five times, the colour of the dye solution had almost disappeared. It was then evaporated to a small bulk on the water-bath, the free hydrocbloric acid being in the first instance eliminated by the addition of sodium acetate. The small quantity of unchanged colouring matter in the evaporated solution was then precipitated by means of a solution of tannic acid in sodium acetate, and the clear filtrate rendered alkaline with a dilute solution of caustic soda. The alkaline solution was then extracted with ether, the ethereal extract dried, and evaporated free from other, when a solid residue was obtained which on crystallisation from a mixture of benzene and light petroleum yielded asym-dimethyl$p$-phenylenediamine in needles melting at $41^{\circ}$ :

0.2191 gave $0.5647 \mathrm{CO}_{2}$ and $0.1755 \mathrm{H}_{2} \mathrm{O} . \quad \mathrm{C}=70.30 ; \mathrm{H}=8.91$. $\mathrm{C}_{8} \mathrm{H}_{12} \mathrm{~N}_{2}$ requires $\mathrm{C}=70.6 ; \mathrm{H}=8.8$ per cent.

The xylene solutions collected from the above experiments were concentrated by distillation, the residue poured into an evaporating 
basin, and freed from xylene on the water-bath. The solid residue, on crystallisation from xylene, yielded dark coloured needles melting at $244^{\circ}$,identical with 3 -dimethylaminophenonaphthoxazone obtained in the former experiment :

$0 \cdot 2115$ gave $17 \cdot 35$ c.c. of nitrogen at $15^{\circ}$ and $775 \mathrm{~mm}$. $\quad \mathrm{N}=9 \cdot 87$. $\mathrm{C}_{10} \mathrm{H}_{14} \mathrm{O}_{2} \mathrm{~N}_{2}$ requires $\mathrm{N}=9 \cdot 7$ per cent.

Formation of 3-Dimethylaminophenonaphthoxazone from Meldola's Blue.

The specimen of Meldola's blue used in this experiment had been specially purified for pathological work by Dr. Grübler, of Leipzig; the following analysis showed it to be pure:

0.2313 gave $18 \cdot 00$ c.c. of nitrogen at $19^{\circ}$ and $770 \mathrm{~mm} . \quad \mathrm{N}=9 \cdot 17$. $\mathrm{C}_{18} \mathrm{H}_{14} \mathrm{ON}_{2}, \mathrm{HCl}$ requires $\mathrm{N}=9 \cdot 0$ per cent.

In order that Meldola's blue may be completely converted into the oxazone it is necessary (as explained on page 329) that oxidation should take place at the same time, or, in other words, that only half of the blue can be converted into the oxazone at the expense of the other half, which becomes thereby reduced to the leuco-base. The process adopted was as follows.

Three grams of pure Meldola's blue were dissolved in 500 c.c. of water, acidified by the addition of 5 c.c. of concentrated hydrochloric acid, and mixed with 100 c.c. of xylene. On shaking the mixture, the xylene remained colourless, showing that no immediate formation of the oxazone takes place on solution in water. On standing, however, and again shaking, the xylene showed traces of fluorescence, indicating that the process takes place slowly at the ordinary temperature. The mixture was heated on the water-bath for twentyfour hours, after which time the xylene solution had become deeply coloured and strongly fluorescent. These facts are in accordance with the behaviour of Meldola's blue when used as a stain. When the solution of the colour is first prepared it does not possess the power of staining fat red in a pathological section, but after boiling for a short time with dilute acids it possesses this property to a marked degree. The xylene solution was separated, and the dye solution, which contained a considerable quantity of brownish insoluble matter, filtered. All attempts made with the object of purifying this precipitate were unsuccessful, since it rapidly oxidised in the air. It was, therefore, again transferred to the dye solution and the whole placed in a large Winchester-quart bottle, which it half filled, and the bottle shaken on the shaking machine for twelve hours. After this time it was noticed that the precipitate had again passed into solution, and the mixture was therefore transferred to the original flask, covered with 100 c.c. of xylene, and heated with constant shaking for eight hours. 
The xylene was then again separated and the dye solution subjected to a further shaking for twelve hours, and once again heated with 100 c.c. of xylene for six hours. The xylene solutions from these experiments were concentrated by distillation, the concentrated solution evaporated free from xylene on the water-bath, and the residue crystallised from xylene. 3-Dimethylaminophenonaphthoxazone obtained in this way melted at $244^{\circ}$, and was in all respects identical with the compound prepared in the previous cases:

0.2358 gave $19 \cdot 35$ c.c. of nitrogen at $15 \cdot 5^{\circ}$ and $774 \mathrm{~mm} . \quad \mathrm{N}=9 \cdot 83$. $\mathrm{C}_{18} \mathrm{H}_{14} \mathrm{O}_{2} \mathrm{~N}_{2}$ requires $\mathrm{N}=9 \cdot 7$ per cent.

It is, of course, impossible by this means to convert the whole of the Meldola's blue into the oxazone, but from the experiments described an amount of 3-dimethylaminophenonaphthoxazone was obtained corresponding with 80 per cent. of the amount of blue taken.

\section{Experiments with Capri-blue GN and Capri-blue GON.}

These colouring matters were experimented on in order to ascertain whether derivatives of phenoxazine containing amino- or substituted amino-groups in the $p$-position to the oxazine nitrogen would behave in the same way as the corresponding derivatives of phenonaphthoxazine. Experiments with the two Capri-blues mentioned above, the formulæ of which are given on page 325 , showed that these colouriug matters did not possess the property of staining fat either when dissolved in water or after boiling for a considerable time with dilute acids. The experiments with xylene bore out these indications, and although after heating an acidified solution of these colouring matters for several days with xylene the xylene solutions became faintly red, yet the amount of substance obtained on evaporating the xylene was too small for further investigation.

My thanks are due to Mr. A. R. Smith, of the Manchester University, for valuable help given during the course of this investigation.

Manchester University. 